\title{
Funktionswörter als Indikatoren für diskursspezifi- sche Argumentationsstrukturen im printmedialen Diskurs über den fünften Sachstandsbericht des Weltklimarates*
}

\author{
Nadine Kammermann (Bern)
}

\begin{abstract}
This study aims to explore how Swiss print media convey climate change to laypeople. Due to the subjects' complexity and scope, its mediation can result in difficulties. A corpus of articles on the IPCC (Intergovernmental Panel on Climate Change) provides an insight into discursive argumentation structures. Corpus-driven collected function word clusters enable to identify argumentative schemes in the media discourse. The qualitative analysis of these argumentative clusters reveals the significance of several actors, particularly administrative and cognitive authorities. Furthermore, prominent are institutional features and differences in scientific and everyday media argumentation norms.
\end{abstract}

\section{$1 \quad$ Einleitung}

Diskurse in Printmedien thematisieren und (re)produzieren kollektives Diskurswissen. Insbesondere bei strittigen Themen ist es lohnenswert, zu betrachten, welches Wissen Printmedien mit welchen (argumentativen) Mitteln vermitteln, was wiederum unmittelbar dazu beiträgt, wie das vermittelte Wissen von den Rezipient*innen wahrgenommen wird. Dies anhand des printmedialen Diskurses über den Klimawandel in der Schweiz zu erörtern, hat zweierlei Gründe: Erstens gibt es bis dato kaum Erkenntnisse zur Berichterstattung über den Klimawandel in der Schweiz ${ }^{1}$ und auch international nur unzureichende Erkenntnisse zum argumentativen Gehalt dieses Diskurses ${ }^{2}$. Zweitens eignet sich gerade der Klimawandel für die Betrachtung von argu-

\footnotetext{
* Die Resultate, die in diesem Artikel vorgestellt werden, entstammen meiner unveröffentlichten Masterarbeit (Kammermann 2014). Entsprechend lassen sich einzelne Teile der Masterarbeit im Artikel wiederfinden. 1 Besio/Pronzini (2010) sowie Ehrensperger (2009) betrachten den printmedialen Diskurs über den Klimawandel in der Schweiz. Peripher beschäftigen sich auch Schmidt/Ivanova/Schäfer (2013), welche das Korpus von Besio/Pronzini (2010) für einen internationalen Vergleich verwenden, sowie Grundmann (2006), der u. a. Artikel der Neuen Zürcher Zeitung für ein entsprechendes deutschsprachiges Korpus verwendet, mit diesem Diskurs. 2 (Massenmediale) Diskurse über den Klimawandel wurden bereits zahlreich verhandelt (Überblicke über diese Themenkomplexe bieten beispielsweise Boykoff (2011) und Neverla/Schäfer (2012)), allerdings oft ohne besondere Berücksichtigung der argumentativen Dimensionen des Diskurses.
} 
mentativen Strukturen, da die massenmediale Vermittlung von klimawandelspezifischem Wissen komplex ist: Annahmen, die auf wissenschaftlichen Grundlagen fussen (s. Kapitel 4.1), werden durch Institutionen (überstaatlichen Vereinigungen, Einzelstaaten oder politische Akteur*innen) in eine praktische Umsetzung überführt. Diese Prozesse sind oft durch normative Aussagen geprägt und emotional; nicht nur wissenschaftliche Grundlagen müssen verortet werden, sondern auch politische Konsequenzen sowie moralische und ethische Implikationen. Fragen nach dem Ausmass sowie den potentiellen Konsequenzen des Klimawandels haben starke gesellschaftliche, politische und ökonomische Auswirkungen. Dementsprechend viele Spezialdiskurse wie beispielswiese Politik oder Wissenschaft, die domänspezifische (argumentative) Eigenschaften aufweisen, gilt es im massenmedialen Diskurs über den Klimawandel miteinander zu verknüpfen. Erschwerend kommt hinzu, dass massenmediale Diskurse für Lai*innen oft die einzige Möglichkeit sind, um mit dem Expert*innenwissen der Spezialdiskurse in Kontakt zu kommen. Dadurch nehmen Medien in diesem Diskurs die prototypische Vermittlerrolle ein. Argumentative Besonderheiten dieser Vermittlerrolle sollen in diesem Artikel näher beleuchtet werden.

Anhand des Fünften Sachstandsberichts des Weltklimarats als Prototext soll in diesem Artikel aufgezeigt werden, dass Funktionswörter als Zugangspunkt zu argumentativen Strukturen für die Analyse innerhalb einer kritischen Diskursanalyse dienen können. Hierfür werden im zweiten Kapitel die diskursanalytischen und argumentativen Grundlagen erarbeitet, um dann im dritten Kapitel die Methode zu erläutern, im vierten Kapitel eine Analyse des massenmedialen Diskurses über den Klimawandel vorzunehmen und den Artikel dann im fünften Kapitel mit einem Fazit zu beschliessen.

\section{Theoretische Zugangspunkte}

\subsection{Diskursanalytische Grundlagen}

Der Diskurshistorische Ansatz (z. B. Reisigl 2011; Reisigl/Wodak 2016; Wodak et al. 2009) eignet sich als kritisch-diskursanalytischer Zugangspunkt zu den Argumentationen eines Diskurses, da er ebenjene als zentrale Bestandteile desselbigen auffasst:

We consider 'discourse' to be:

- a cluster of context-dependent semiotic practices, that are situated within specific fields of social action;

- socially constituted and socially constitutive;

- related to a macro-topic;

- linked to argumentation about validity claims, such as truth and normative validity involving several social actors with different points of view.

(Reisigl/Wodak 2016: 27)

Im Rahmen dieses Artikels soll keine vollständige Analyse im Sinne des Diskurshistorischen Ansatzes vorgenommen werden, sondern stattdessen betrachtet werden, wie das Makrothema Fünfter Sachstandsbericht des IPCC in Zeitungen der deutschsprachigen Schweiz argumentativ situiert wird. Somit liegt der Fokus auf dem letzten Punkt, den Reisigl/Wodak (ibd.) anführen. Der kontextuellen und diskursiven Einbettung wird in den Kapiteln 4.1 und 4.2 teilweise 
Rechnung getragen, indem eine Verortung der institutionellen Rahmenbedingungen des IPCC und deren mögliche Auswirkungen auf den Diskurs vorgenommen wird.

\subsection{Argumentation in Diskursen}

Argumentationen weisen im Alltag selten formallogische - streng induktive oder deduktive Eigenschaften auf. In der Regel handelt es sich stattdessen um sogenannte Enthymeme. Solche setzen einen strittigen Sachverhalt voraus, der durch Meinungsbildung über eine weniger strittige (oder unstrittige) These in eine unstrittige Konklusion überführt wird. Hierfür gilt das kollektive Wissen der Gesprächsteilnehmer*innen als Basis. Diese Überführung basiert auf einem quasilogischen, probabilistischen Schluss (cf. Ottmers 2007). Dementsprechend grenzt sich ein alltägliches Argument auch in den folgenden fünf Punkten von einem formallogischen Argument ab (ibd.: 75):

1. Die formale Struktur ist nicht festgelegt.

2. Teile des Enthymems können implizit bleiben.

3. Die Überführung in eine unstrittige These erfolgt über einen Plausibilitätsschluss.

4. Das Argument muss unstrittig sein.

5. Enthymeme beruhen auf Schlussverfahren, die in Topoikatalogen (z. B. Topik von Aristoteles) beschrieben werden können.

Es gibt eine Reihe von einschlägiger Literatur, die sich mit alltagslogischer Argumentation beschäftigt. ${ }^{3}$ Ich werde mich im Folgenden an der in Reisigl/Wodak (2016: 35) vorgeschlagenen Definition und Terminologie orientieren: ,'Topoi' [...] can be understood as parts of argumentation that belong to the required premises. They are formal or content-related warrants or "conclusion rules'. As such, they connect the argument(s) with the conclusion, the claim."

Argumentationsstrukturen werden üblicherweise über ihre warrants, Schlussregeln, klassifiziert und können grob in alltagslogische Schlussverfahren und konventionalisierte Schlussverfahren unterteilt werden. Letztere umfassen beispielsweise normative Prämissen, moralische Grundsätze oder Klischees. Alltagslogische Topoi können in allgemeine (Wenn schon p, dann erst recht q.) und spezifische (materiale) Topoi (Wenn weniger Autos auf den Strassen unterwegs sind, dann kann der $\mathrm{CO}_{2}$-Ausstoss reduziert werden.) unterteilt werden (vgl. Ottmers 2007: 88-92). Die Betrachtung von materialen Topoi gewinnt für den Diskurs über den Klimawandel insbesondere an Relevanz, wenn man "the observation [folgt] that argumentation is alway topic-related and field-dependent (i.e. depending on the configuration of social domains, dicsiplines, theories, etc.)" (Reisigl 2014: 77), da hier mehrere Spezialdiskurse verknüpft sind.

Die Betrachtung dieser spezifischen Topoi bietet Auskunft darüber, welche Propositionen im Rahmen von Geltungsansprüchen der Wahrheit und der (normativen) Richtigkeit im Diskurs verhandelt werden. Es lässt sich aufzeigen, welches Wissen bereits als unstrittig im Diskurs etabliert ist und welche Propositionen erst noch etabliert werden müssen.

Die Argumentation eines Diskurses zu erfassen, ist unabhängig von der gewählten theoretischen Einbettung sowie den methodologischen Grundlagen äusserst zeitaufwändig und mit

3 Beispielsweise Perelman/Olbrechts-Tyteca 1958/2004, Kienpointner 1992, Kopperschmidt 1989, van Eemeren/Grootendorst 2004. 
Restriktionen verbunden; Wengeler (2003) behilft sich beispielsweise damit, dass er die Analyse auf 38 im Voraus gewonnene, spezifische Topoi beschränkt. Über diese Restriktion der betrachteten Topoi ist somit eine Analyse auch über eine Vielzahl an Texten möglich. Andere Autor*innen wählen eher quantitative Zugänge (oft über Argumentationsindikatoren; z. B. Kindt 2008 oder Bubenhofer 2008; einen verhältnismässig umfassenden theoretischen Zugang zu argumentativen Indikatoren für das Englische bietet van Eemeren/Houtlosser/Snoeck Henkemans 2007). Allerdings lassen sich mit rein quantitativen Ansätzen die unter der sprachlichen Oberfläche gelagerten Teile der Argumentationsstruktur nicht erfassen. Somit bleiben sowohl qualitative als auch quantitative Zugänge sehr oft auf einer exemplarischen Ebene. Um mit den Vor- und Nachteilen der beiden methodischen Grundsätzen adäquat umzugehen, soll in diesem Artikel eine Synergie zwischen qualitativen und quantitativen Methoden angestrebt werden: Funktionswörter werden als mögliche Zugangspunkte skizziert, um die Argumentationsstrukturen im Diskurs quantitativ erfassen und anschliessend qualitativ auswerten zu können.

\subsection{Funktionswörter als Zugang zur Argumentation}

Funktionswörter (auch: Synkategoremata oder closed-class words) ${ }^{4}$ finden korpus- und diskursanalytisch oft wenig Beachtung, obwohl ihre Betrachtung gerade in Hinblick auf eine kritische Diskursanalyse einen Erkenntnisgewinn darstellen kann, da

The fact that much CDA work is concerned with exploring aspects of discourse which are not immediately obvious to the casual reader (thus rendering their effects more powerful ideologically) means that function words must be worth considering in corpus-assisted critical discourse analysis.

(Pearce 2014: 24)

Groom (2010: 63) schlägt ebenfalls vor, Funktionswörter als quantitativen Startpunkt einer korpuslinguistischen Diskursanalyse zu verwenden:

For corpus-driven discourse analysis, the crucial distinction is not between ontologically different categories of keywords [= Syn- und Autosemantika] (aboutness versus style, lexical versus grammatical, content versus function, etc.), but between methodologically different ways of looking at keywords; specifically, as endpoints of quantitative analysis on the one hand [= Autosemantika], or as starting points for qualitative analysis on the other [= Synsemantika].

(ibd.: 63)

Indem Funktionswörter als Zugang gewählt werden, kann eine grosse Menge an repräsentativen Stellen im Text erfasst und für eine qualitative Analyse zugänglich gemacht werden. Im Folgenden wird in aller Kürze der quantitative Zugang erläutert, die nachfolgenden Kapitel sind der qualitativen Analyse gewidmet.

\footnotetext{
4 „Funktionswörter“ ist eine „Bezeichnung für sprachliche Elemente, die primär grammatische (anstelle von lexikalischer) Bedeutung tragen, weshalb sie auch als Synsemantikum bezeichnet werden: Sie erfüllen vor allem syntaktisch-strukturelle Funktionen wie z. B. Artikel, Pronomen, Präposition, Konjunktion“ (Bußmann 2002: 232).
} 


\section{$3 \quad$ Korpusaufbau und analysierte Einheiten}

Das Korpus besteht aus 125 Artikeln aus Printmedien der deutschsprachigen Schweiz, die vom 15. September 2013 (eine Woche vor Veröffentlichung des ersten Teils des Fünften Sachstandsberichts) bis zum 12. Juni 2014 (zwei Monate nach Veröffentlichung des dritten und letzten Teils des Fünften Sachstandsberichts) publiziert wurden und die das Suchwort IPCC enthalten. Artikel, welche eine oder mehrere der folgenden Eigenschaften aufwiesen, wurden aus dem Korpus ausgeschlossen:

- Meldungen von Nachrichtenagenturen

- oder Veranstaltungskalendern,

- $\quad$ Leserbriefe und

- $\quad$ Artikel mit IPPC als Akronym mit anderer Bedeutung.

Wie von Groom (2010) vorgeschlagen, bildeten Funktionswörter den quantitativen Zugang zu den zu analysierenden Einheiten im Korpus. Ausgehend von den achtzehn Funktionswörtern unter den zwanzig häufigsten Wortformen im Korpus ${ }^{5}$ wurden kontinuierliche 3- bis 5-Gramme (cf. Bubenhofer 2008), die entweder mit dem Funktionswort beginnen oder enden (zum Beispiel in der Atmosphäre oder heisst es in) erfasst. Mittels eines $\chi^{2}$-Tests ( $\mathrm{p}=0,001, \chi^{2}=10,3$; cf. Oakes 1998) wurden die relevanten Einheiten ermittelt. Die aus diesem Schritt resultierenden 148 Textclusters sowie deren Kontexte dienten als Ausgangspunkt für die qualitative Analyse der Argumentationsstrukturen, die in Kapitel 4.3 vorgestellt wird. In den zuvor folgenden Kapiteln sollen einige Besonderheiten des medialen Diskurses über die Berichte des IPCC erörtert werden.

\section{$4 \quad$ Resultate}

\subsection{Der IPCC als zentrale Institution im Diskurs}

Die Summaries for Policymakers ${ }^{6}$ des Fünften Sachstandberichts des IPCC (Intergovernmental Panel on Climate Change) bildeten die Grundlage für einen Mediendiskurs, der über Monate anhielt. Dabei wurde sowohl über den Inhalt der Berichte als auch über die Berichte selbst diskutiert. Die drei Zusammenfassungen erinnern somit an den Vortrag von Peter Sloterdijk, der im Rahmen der sogenannten Sloterdijk-Debatte ebenfalls eine enorme Medienresonanz auslöste (ausführlich zur Sloterdijk-Debatte sowie zum Konzept des Prototextes cf. Steinseifer 2005). Um den Diskurs adäquat erfassen zu können, sollen deshalb zunächst die Beschaffenheit sowie der Inhalt der SPM umrissen werden, da diese Prototexte für die Berichterstattung darstellen und bestimmte institutionelle Besonderheiten aufweisen.

\footnotetext{
${ }^{5}$ Die folgende Auflistung zeigt die zwanzig häufigsten Tokens im Korpus mit der absoluten Frequenz in Klammern. Fett hervorgehoben sind die für die Analyse relevanten Funktionswörter: die (2960), der (2701), und (1372), in (1232), den (958), das (814), ist (779), zu (741), von (684), des (667), sich (651), es (583), nicht (575), auf (523), für (523), im (508), dass (495), mit (482), als (468), IPCC (455).

${ }^{6}$ Im Folgenden wird das übliche Akronym SPM für Summary for Policymaker verwendet.
} 
Der IPCC (im Deutschen häufig auch als Weltklimarat oder UNO-Klimarat bezeichnet) unterliegt dem United Nations Environment Programme sowie der World Meteorological Organization und wurde 1988 gegründet. Das Ziel dieser Organisation ist es, die aktuellen Erkenntnisse bezüglich des Klimawandels und des Klimasystems zu diskutieren und zu bündeln.

In Abständen von mehreren Jahren werden umfassende Berichte veröffentlicht, die den aktuellen Wissensstand zum Klimawandel aufzeigen und diskutieren. Die Reflexion des aktuellen Wissensstandes findet in freiwilliger und unentgeltlicher Zusammenarbeit von Forscher*innen unterschiedlichster Fachbereiche statt (cf. "Review of IPCC Processes and Procedures"). Wissenschaftler*innen, die dem IPCC angehören, müssen deshalb folgende Bedingungen erfüllen:

- Experts who have significant expertise and/or publications in particular areas covered by the Report.

- Experts nominated by governments as Coordinating Lead Authors, Lead Authors, contributing authors or expert reviewers as included in lists maintained by the IPCC Secretariat.

- Expert reviewers nominated by appropriate organisations.

(IPCC 1999-2008: 6)

Diese diskursiven Rahmenbedingungen haben bereits signifikante Auswirkungen auf eines der häufigsten Argumente im Diskurs: das Autoritätsargument. Hierfür führt Walton (1997: 223) folgende kritischen Fragen an:

1. Expertise question: How credible is $\mathrm{E}$ as an expert source?

2. Field question: Is $\mathrm{E}$ an expert in the field that $\mathrm{A}$ is in?

3. Opinion question: What did $\mathrm{E}$ assert that implies A?

4. Trustworthiness question: Is E personally reliable as a source?

5. Consistency question: Is A consistent with what other experts assert?

6. Backup evidence question: Is A's assertion based on evidence?

Das im Zitat aufgeführte Auswahlverfahren sowie die unentgeltliche und freiwillige Mitarbeit führen dazu, dass sich die Mehrheit dieser kritischen Fragen bereits durch die Zugehörigkeit zum IPCC beantworten lässt. Die Akezptabilität dieser für den Diskurs wichtigen Argumentationsschemata resultiert somit bereits zu einem grossen Teil aus der Zugehörigkeit zum IPCC, was die Funktion der Berichte des IPCC als Prototexte stärkt.

Sowohl die Forschenden als auch deren Berichte sind in drei Arbeitsgruppen gegliedert:

- Arbeitsgruppe I "aims at assessing the physical scientific aspects of the climate system and climate change" ("Working Group I: The Physical Science Basis").

- Arbeitsgruppe II "assesses the vulnerability of socio-economic and natural systems to climate change, negative and positive consequences of climate change, and options for adapting to it" ("Working Group II: Impacts, Adaptation, and Vulnerability").

- Arbeitsgruppe III "assess the costs and benefits of different approaches to mitigation and consider the available instruments and policy measures to drive and deliver these approaches" ("Working Group III: Mitigation of Climate Change"). 
Diese Arbeitsgruppen erstellen im Rahmen eines klar strukturierten Prozesses je einen Teilbericht (Assessment Report). In enger Zusammenarbeit mit teilnehmenden Regierungen werden anschliessend die dazugehörigen SPM erarbeitet und an einer Versammlung, an der sowohl Klimatolog*innen als auch Politiker*innen anwesend sind, verabschiedet. In dem betrachteten Zeitraum (September 2013 bis Juni 2014) wurden die SPM des Fünften Sachstandsberichts vorgestellt. ${ }^{7}$

Die transparente Erstellung von Berichten sowie die Auswahl der Expert*innen spiegeln sich auch im Umgang mit Studien und den aus Prognosen resultierenden Unsicherheiten wider. Als entsprechende Leitlinie für die SPM gilt Mastrandrea et al. (2010). Die darin enthaltene Notation wird jeweils zu Beginn der drei Berichte erklärt, da sie für das Verständnis der Texte elementar ist. Sie soll hier an folgenden Beispielen aufgezeigt werden:

- Proxy and instrumental sea level data indicate a transition in the late 19th to early 20th century from relatively low mean rates of rise over the previous two millennia to higher rates of rise (high confidence (A)). It is likely (B) that the rate of global sea level rise has continued to increase since the early 20 th century. $\{3.7,5.6,13.2\}$

- It is very likely that the mean rate of global averaged sea level rise was 1.7 [1.5 to 1.9] mm yr ${ }^{-1}$ between 1901 and 2010,2.0 (D) [1.7 to 2.3] (C) $\mathrm{mm} \mathrm{yr}^{-}$between 1971 and 2010, and 3.2 [2.8 to 3.6] $\mathrm{mm} \mathrm{yr}^{-1}$ between 1993 and 2010. Tide-gauge and satellite altimeter data are consistent regarding the higher rate of the latter period. It is likely that similarly high rates occurred between 1920 and 1950. \{13.3\} (E)

(Stocker et al. 2013: 11; Buchstaben in Klammern durch die Autorin)

Die Sicherheit einer Aussage wird über das Vertrauen (confidence) der Forscher*innen beschrieben, welches sich aus ,the type, amount, quality, and consistency of evidence [...] and the degree of agreement" (Mastrandrea et al. 2010: 2) zusammensetzt. In den Texten wird dies folgendermassen angegeben: very low c., low c., medium c., high $c$. und very high c. (A). Falls möglich wird eine statistische Wahrscheinlichkeit angegeben. Diese ist nach folgenden Kriterien aufgeschlüsselt und ebenfalls kursiv hervorgehoben: virtually certain $=99-100 \%$, very likely $=90-100 \%$, likely $=66-100 \%$, unlikely $=0-33 \%$, very unlikely $=0-10 \%$, exceptionally unlikely $=0-1 \%$, extremly likely $=95-100 \%$, more likely than not $=50-100 \%$ und extremely unlikely $=0-5 \%$ (B). Der Bereich in den eckigen Klammern umfasst $90 \%$ aller möglichen Werte (C). Der wahrscheinlichste Wert wird, falls verfügbar, davor angegeben (D). In geschweiften Klammern wird angeführt, in welchen Kapiteln der Assessment Reports sich die vollständigen Informationen zum jeweiligen Abschnitt befinden (E) (cf. ibd.). Durch dieses System lässt sich überprüfen, wie valide ein Sachverhalt ist, ob er statistisch sicher belegt wurde (oder sich überhaupt statistisch belegen lässt), ob sich die Forscher*innen der Arbeitsgruppe bezüglich dieses Sachverhalts einig sind und über die Referenzen sind die Stellen mit den ausführlichen Informationen zugänglich.

\footnotetext{
${ }^{7}$ Vom 23. bis 26. September 2013 wurde in Stockholm der Bericht der Arbeitsgruppe I (cf. Stocker et al. 2013) vorgestellt, der Bericht der Arbeitsgruppe II (cf. Field et al. 2014) schloss vom 25. bis 29. März 2014 in Yokohoma an und der finale Bericht der Arbeitsgruppe III folgte wenig später vom 7. bis 11. April 2014 (cf. Edenhofer et al. 2014) in Berlin.
} 
Solche Ausdrücke wie confidence oder unlikely, die der Markierung von institutionell geregeltem Umgang mit Statistiken und Unsicherheiten bezüglich Prognosen dienen, sind oft die gleichen, die alltagssprachlich für epistemische Modalität verwendet werden und zu einem entsprechenden Geltungsanspruch der Wahrheit führen können. Dies ist insbesondere der Fall, wenn solche Aussagen direkt in den Zeitungsartikeln übernommen werden ohne die zugrundeliegende Methodik zu erläutern. Dadurch erscheinen die Propositionen weniger investigativ belegt und somit strittiger als sie tatsächlich sind.

Da sich die SPM an Aussenstehende richten, werden wichtige Fachbegriffe, Konzepte und Modelle entweder an den jeweiligen Stellen (so bei der Arbeitsgruppe I) oder aber in Form einer Liste (Arbeitsgruppe II) für wissenschaftliche Lai*innen in verständlicher Weise zugänglich gemacht.

\subsection{Diskursübergreifende Betrachtung}

Die Betrachtung der N-Gramme lässt bereits Rückschlüsse auf relevante Konstruktionen im Diskurs zu. Die durch Funktionswörter markierten Stellen im Diskurs scheinen sich auf einige, wenige Aspekte zu fokussieren. Ein Indiz dafür ist, dass sich die N-Gramme in die folgenden Kategorien einordnen lassen:

- Lokative Angaben (z. B. in der Atmosphäre, auf den Philippinen)

- Temporale Angaben (z. B. in den letzten 15 Jahren, bis Ende des)

- Personen- und institutsbezogene Angaben (z. B. Co-Leiter der, von der ETH)

- Angaben zum IPCC (z. B. den ersten Teil, der Arbeitsgruppe I des IPCC)

- Klimawissenschaftliche Angaben (z. B. die globale Erwärmung, das $\mathrm{CO}_{2}$-Budget)

- Fakten und Zahlen (z. B. zwei Drittel des, von 18 bis 59 Zentimetern)

- Syntaktische Strukturen (z. B. wenn nicht die, und in der)

Die wissenschaftlichen Angaben beschränken sich mehrheitlich auf das thematische Feld der Arbeitsgruppe I. Die syntaktischen Strukturen wurden in der folgenden Analyse nicht mehr berücksichtigt, da sie nicht unmittelbar diskursspezifisch sind.

Aus der Betrachtung der Kontexte der N-Gramme lassen sich bereits bestimmte diskursive Makrostrukturen (cf. zur Terminologie Kindt 2008) ableiten:

- Aus der Klimaentwicklung der Vergangenheit lässt sich (nicht) auf zukünftige Klimaentwicklungen schliessen.

- Das Kyoto-Protokoll läuft aus. Ein neuer Klimavertrag für die Zeit nach 2020 muss erstellt werden. Es ist unklar, wie der Vertrag kontrolliert werden soll. Alle Länder sollen zukünftig Verantwortung tragen, auch die Schwellenländer.

- Der Mensch ist für den Klimawandel verantwortlich.

- Die Berichte des IPCC bilden die Grundlage für die zukünftige Klimapolitik. Die Institution bildet eine Schnittstelle zwischen Wissenschaft und Politik.

- Es muss jetzt gehandelt werden, da es sonst zu spät ist. Je länger man wartet, desto schwieriger und kostspieliger wird es, das 2-Grad-Ziel zu erreichen. 
- Im Vergleich zur vorindustriellen Zeit hat sich die Temperatur um durchschnittlich $0,8^{\circ} \mathrm{C}$ erwärmt, und es wurden 3000 Milliarden Tonnen $\mathrm{CO}_{2}$ in die Luft ausgestossen. Dies sind zwei Drittel des $\mathrm{CO}_{2}$-Budgets, um das 2-Grad-Ziel zu erreichen.

- In den letzten fünfzehn Jahren fand eine Klimastagnation statt.

- Wenn bis zum Zeitpunkt X die Emissionen nicht um Y reduziert werden, kann das 2-GradZiel nicht erreicht werden. Wird das 2-Grad-Ziel nicht erreicht, so führt dies zu Problemen, die der Mensch nicht lösen kann.

Diese Makrostrukturen haben situativ unterschiedliche Funktionen; sie repräsentieren teilweise vollständige Topoi, aber teilweise auch nur (implizite oder explizite) Prämissen oder Konklusionen. So kann die Aussage Der Mensch ist (nicht) für den Klimawandel verantwortlich eine Konklusion sein oder aber eine Prämisse dafür, etwas (nichts) gegen den Klimawandel zu unternehmen. Oft manifestieren sich diese Makrostrukturen, indem eine entsprechende Aussage durch eine Expert*innenmeinung legitimiert wird: „Der neuste Bericht des UNO-Wissenschaftsrats macht deutlich, dass der Mensch als Hauptfaktor die Klimaerwärmung verursacht" (Neue Luzerner Zeitung 11.01.2014; Hervorhebungen durch die Autorin). „Die Autoren erläutern darin [= IPCC-Bericht], dass sich das Ausmass des vom Menschen angetriebenen Klimawandels wohl nur noch mit grossen Reformen so weit mindern liesse, dass keine bedrohlichen Folgen zu erwarten sind“ (Neue Zürcher Zeitung 15.04.2014; Hervorhebungen durch die Autorin). Dies ist ein erstes Indiz für die in 4.1 erarbeitete These, dass Autoritätsargumente im Zentrum des Diskurses stehen.

\subsection{Argumentationsschemata}

\subsubsection{Kausale Schemata}

Auf der Ebene der Berichte des IPCC sind Kausalschemata essentiell. Sie thematisieren Ursache(n) und Wirkung(en) des Klimawandels. Dies ist notwendig, um Anpassungs- und Minderungsmassnahmen erarbeiten zu können. Die erste Arbeitsgruppe beschäftigt sich hauptsächlich mit den physikalischen Grundlagen des Klimawandels: "The rate of sea level rise since the mid-19th century has been larger than the mean rate during the previous two millennia (high confidence). Over the period 1901 to 2010 , global mean sea level rose by 0.19 [0.17 to 0.21] m" (Stocker et al. 2013: 11; Hervorhebungen im Original). Die zweite Gruppe erörtert die ökologischen und gesellschaftlichen Folgen des Klimawandels: 'Due to sea level rise projected throughout the 21st century and beyond, coastal systems and low-lying areas will increasingly experience adverse impacts such as submergence, coastal flooding, and coastal erosion (very high confidence)" (Field et al. 2014: 17; Hervorhebungen im Original). Die dritte Arbeitsgruppe thematisiert mögliche Anpassungs- und Minderungsmassnahmen sowie deren Kosten: "Important options for mitigation in waste management are waste reduction, followed by re-use, recycling and energy recovery (robust evidence, high agreement)" (Edenhofer et al. 2014: 24; Hervorhebungen im Original).

Betrachtet man die Berichte der drei Arbeitsgruppen gemeinsam, so ergibt sich eine Kette aus Ursachen, Wirkungen und Massnahmen. 
Die Überführung dieser kausalen Argumentationsschemata des IPCC in Zeitungsartikel wird durch zwei Sachverhalte erschwert:

1. Um die zukünftigen Folgen des Klimawandels abzuschätzen, sind Prognosen notwendig. Aus ihnen resultieren (statistische) Unsicherheiten.

2. Der Kausalitätsbegriff der Alltagssprache unterscheidet sich von demjenigen der Wissenschaftssprache.

Die Ceteris-Paribus-Klausel der Alltagslogik steht derjenigen der Wissenschaft - und insbesondere des Experimentes - gegenüber (cf. Kienpointner 1992: 331), da in letzterer der Kausalitätsbegriff sehr eng gefasst wird. Im Gegensatz dazu wird "in Alltagsargumentationen [...] oft stillschweigend monokausal argumentiert, indem eben eine Ursache als die entscheidende bzw. im speziellen Kontext einzig wichtige angesehen wird" (ibd.: 332). Diese oft auch den Lai*innen- und Mediendiskursen inhärente Monokausalität der Alltagsargumentation steht eine wissenschaftliche Argumentation gegenüber; Polykausalität ist hier die Regel.

\section{(1) Einfache, monokausale Argumentationsstruktur}

Übermässig viel FCKW wird in die Atmosphäre emittiert.

Wenn übermässig viel FCKW in die Atmosphäre emittiert wird, vergrössert sich das Ozonloch.

Das Ozonloch vergrössert sich.

Komplexe Zusammenhänge in den Klimawissenschaften lassen sich nur selten auf das Format "Ursache $\rightarrow$ Wirkung" wie in (1) reduzieren. Im Gegenteil handelt es sich meist um kausale Argumentationsketten:

Ursache $(\mathrm{n})_{1} \rightarrow$ Wirkung $(\mathrm{en})_{1}=\operatorname{Ursache}(\mathrm{n})_{2} \rightarrow$ Wirkung $(\mathrm{en})_{2}=\operatorname{Ursache}(\mathrm{n})_{3}$ usw.

Eine solche polykausale Argumentationskette findet sich bereits zu Beginn der Zusammenfassung der ersten Arbeitsgruppe: „The atmosphere and ocean have warmed, the amounts of snow and ice have diminished, sea level has risen, and the concentrations of greenhouse gases have increased" (Stocker et al. 2013: 4).

\section{(2) Ebene $1^{8}$}

Die Treibhausgaskonzentration hat sich erhöht.

(Wenn sich die Treibhausgaskonzentration erhöht, erwärmt sich die Atmosphäre.)

Die Atmosphäre erwärmt sich.

(3) Ebene 2

a) Die Atmosphäre hat sich erwärmt.

(Wenn sich die Atmosphäre erwärmt, schmelzen Schnee und Eis.)

Schnee und Eis schmelzen.

b) Die Atmosphäre hat sich erwärmt.

\footnotetext{
${ }^{8}$ Implizite (und entsprechend) rekonstruierte Prämissen oder Konklusionen werden in den Darstellungen mit Klammern versehen. In der ersten Zeile wird die Hauptprämisse genannt, in der zweiten folgt der diskursspezifische Topos - die Schlussregel. Die Konklusion folgt als letztes Element einer einzelnen Argumentationsstruktur.
} 
(Wenn sich die Atmosphäre erwärmt, erwärmen sich die Ozeane.)

Die Ozeane erwärmen sich.

(4) Ebene 3

Die Ozeane haben sich erwärmt.

(Wenn sich die Ozeane erwärmen, schmelzen Schnee und Eis.)

Schnee und Eis schmelzen.

\section{(5) Ebene 4}

Schnee und Eis schmelzen.

(Wenn Schnee und Eis schmelzen, steigt der Meeresspiegel.)

Der Meeresspiegel steigt.

Werden die Schemata grafisch dargestellt, ergibt sich folgendes Bild:

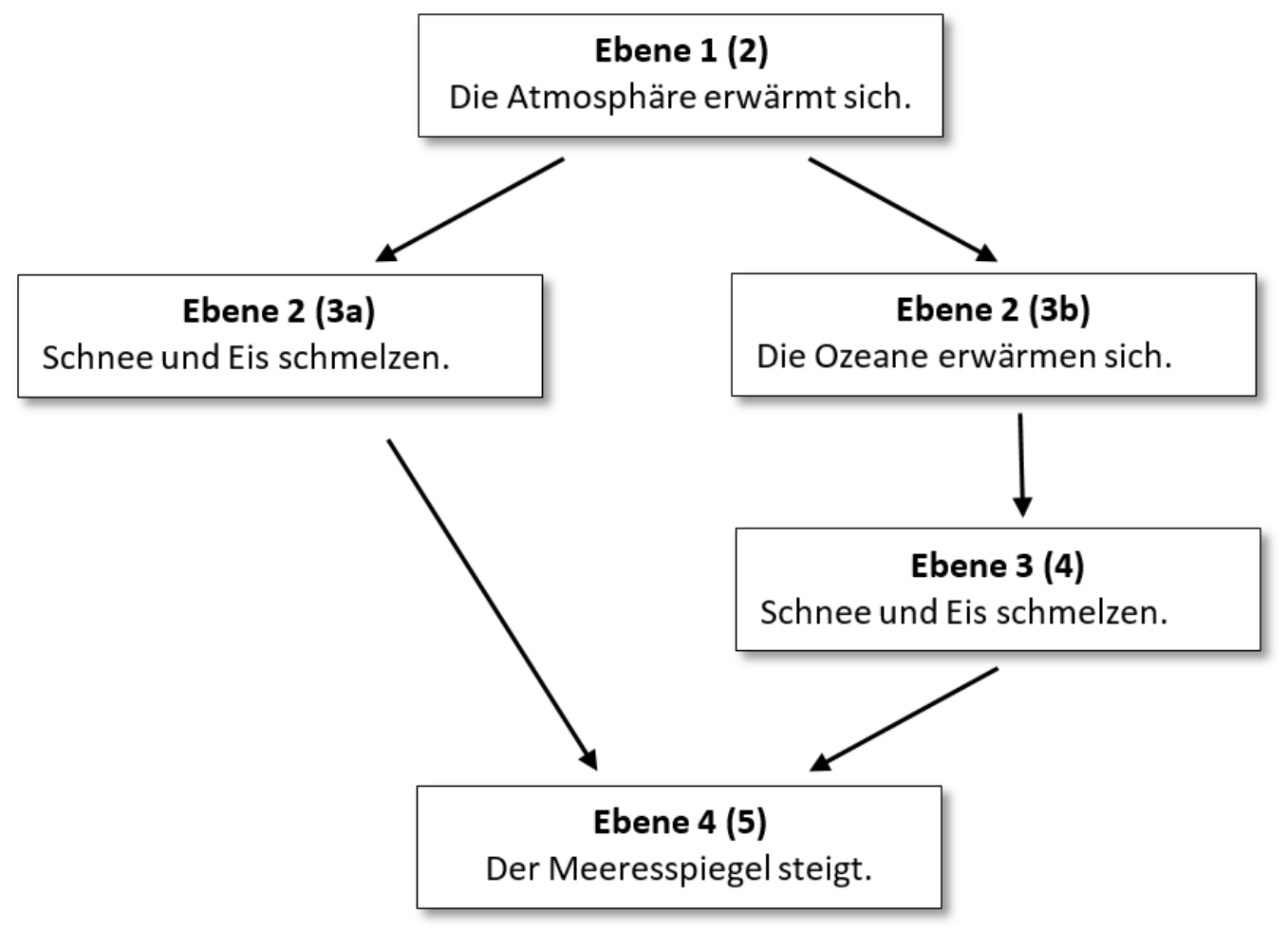

Abbildung 1 Graphische Darstellung der Kausalketten (2) bis (5). Die Konklusion steht jeweils unter der Ebenenbezeichnung.

Nicht nur Ursachen und Wirkungen stehen hier in einem für einen alltagssprachlichen Laiendiskurs schwierigen Verhältnis, sondern die Kausalkette (Abbildung 1) entspricht auch nicht der Reihenfolge der Propositionen im Text; die Hauptkonklusion (Ebene 4) entspricht der zweiten Proposition, die Hauptprämisse „Die Treibhausgaskonzentration hat sich erhöht“ steht gar an letzter Stelle. 


\subsubsection{Autoritätsschemata}

Die Gültigkeit wissenschaftlicher Argumentation, wie sie in 4.3.1 vorgestellt wurde, lässt sich durch Lai*innen kaum beurteilen. Deshalb werden die Propositionen oft über Autoritätsschemata legitimiert. Solche sind in der Regel abduktiv, da sich Autoritäten selten in abgeschlossenen Welten bewegen, dementsprechend beinhaltet ein Argument from Authority "a statistical generalization about the number of times, or percentage of times, that what the authority has said is true" (Walton 1997: 100). Im institutionellen Rahmen des IPCC wird dieser statistische, abduktive Vorgang zusätzlich durch die in Kapitel 4.1 erläuterten Verfahren gesichert, indem nicht nur ein Individuum, sondern eine ganze Gruppe von Expert*innen befragt wird. Dadurch wird die Proposition investigativer belegt als lediglich durch eine Fachstimme.

Entsprechend zentral sind in nahezu allen Zeitungsartikeln Stellen wie (6), die eine Proposition im Klimadiskurs durch eine Expert*innenmeinung legitimieren:

\section{(6) Expert Opinion Ad Populum Argument}

(Der IPCC ist eine Expert*innengruppe der Klimawissenschaften und verfasst die SPM.)

Die Treibhausgasemissionen hätten sich verstärkt, heisst es in der SPM.

(Die Treibhausgasemissionen haben sich verstärkt.)

Die Treibhausgasemissionen hätten sich - trotz der Anstrengungen zu ihrer Reduzierung - verstärkt, heisst es in der Zusammenfassung des Berichts für politische Entscheidungsträger.

(St. Galler Tagblatt 14.04.2014)

Ein Autoritätsargument kann sich entweder auf einzelne Expert*innen (Argument from Expert Opinion) oder aber auf Expert*innengruppen (Expert Opinion Ad Populum Argument) beziehen. Die befürwortenden Artikel stützen sich mehrheitlich auf Reto Knutti und Thomas Stocker als Einzelpersonen sowie auf den IPCC als die institutionelle Expert*innengruppe der Klimawissenschaften. Die Einzelpersonen werden oft zusätzlich durch ihre Funktion als Klimato$\log$ *innen und durch ihre Institutszugehörigkeit legitimiert: „, Die Schweiz hat eine lange Tradition der Beiträge zum IPCC،, sagte Thomas Stocker von der Universität Bern, Ko-Leiter der Arbeitsgruppe, die den ersten Teil des 5. Berichts des Weltklimarats verfasst hat" (St. Galler Tagblatt 27.09.2013).

Klimawandelskeptische Artikel nehmen mehrheitlich auf Nigel Lawson Bezug, der als Politiker kein Experte der Klimawissenschaften ist. Die kritischen Fragen von Walton (1997: 223) lassen sich deshalb nicht (oder nur unbefriedigend) beantworten; eine entsprechende Thematisierung findet allerdings im Rahmen der Artikel kaum statt.

Als kognitive Autorität ${ }^{9}$ legt der IPCC die klimawissenschaftlichen Grundlagen fest. Politiker*innen, Staaten oder andere Akteur*innen und Institutionen setzen diese Grundlagen als

\footnotetext{
9 Walton (1997: 76-79) differenziert zwischen kognitiver (epistemischer, de facto) und administrativer (deontischer, de jure) Autorität. Eine kognitive Autorität ist ein/e Expert/in in einem gewissen Feld und verfügt über das entsprechende Wissen. Die administrative Autorität hat hingegen das Recht, Regeln festzulegen und zu entscheiden "what should be done in certain types of situations, based on an invested office, or an official or recognized position of power" (ibd.: 78).
} 
administrative Autoritäten im Rahmen der Klimapolitik um (beispielsweise durch die Erstellung des Kyoto-Protokolls). Dieser Zweiteilung wird auch in den Artikeln Rechnung getragen, explizit beispielsweise an folgender Stelle: „Die Berichte des 1988 gegründeten Weltklimarates (IPCC) mit Sitz in Genf sollen die Basis für eine wissenschaftlich fundierte Klimapolitik schaffen“ (Neue Zürcher Zeitung 28.09.2013).

\subsubsection{Schemata des Practical Reasoning}

Somit resultieren aus dem Dialog und der Zusammenarbeit von kognitiver und administrativer Autorität im Idealfall Handlungsanweisungen (oft in Form von Practical Reasoning) der administrativen Autorität: "Practical Reasoning is a kind of inference that leads to action in a particular situation and is based on what the agent knows (knowledge base) in a situation as he (she, or it) sees the situation, which may be fluid and uncertain (in common realistic cases of acting on the basis of expert advice)" (Walton 1997: 120).

In den Artikeln wird gewissermassen ein (teils fiktiver, teils existierender) Dialog zwischen administrativer und kognitiver Autorität aufgezeigt, indem die Frage erörtert wird, wie die zukünftige Klimapolitik (der administrativen Autorität) aufgrund der wissenschaftlichen Grundlagen (der kognitiven Autorität) aussieht (oder aussehen soll):

\section{(7) Expert Opinion Ad Populum Argument}

(Der IPCC ist eine Expert*innengruppe der Klimawissenschaften und verfasst die SPM.)

Der IPCC hat in seinem neuesten Bericht aufgezeigt, dass das $\mathrm{CO}_{2}$-Budget für das 2-Grad-Ziel in 18 Jahren aufgebraucht ist.

(Das $\mathrm{CO}_{2}$-Budget für das 2-Grad-Ziel ist in 18 Jahren aufgebraucht.)

In 18 Jahren ist es so weit. Dann hat die Menschheit seit Beginn der Industrialisierung knapp 3000 Milliarden Tonnen $\mathrm{CO}_{2}$ in der Atmosphäre entsorgt. Damit hat sie ihr $\mathrm{CO}_{2}$ Budget aufgebraucht, wenn sie die Klimaerwärmung auf zwei Grad begrenzen will, wie der Weltklimarat der Vereinten Nationen (IPCC) in seinem neusten Bericht aufgezeigt hat.

(St. Galler Tagblatt 11.11.2013)

\section{(8) *Practical Inference}

Die Erderwärmung muss auf $2^{\circ} \mathrm{C}$ begrenzt werden.

Um die Erderwärmung auf $2{ }^{\circ} \mathrm{C}$ zu begrenzen, darf nur eine gewisse Menge an $\mathrm{CO}_{2}$ in die Luft ausgestossen werden.

Die Menge an ausgestossenem $\mathrm{CO}_{2}$ muss reduziert werden.

Dieser neue Vertrag soll das Kyoto-Protokoll ersetzen und dann nicht nur für die Industriestaaten, sondern für alle Länder verbindliche Emissionsziele vorgeben. Das $\mathrm{CO}_{2-}$ Budget werde dabei aber keine Rolle spielen, sagt Christiana Figueres, Chefin der UNOKlimaverhandlungen. ,Ich glaube nicht, dass das möglich ist. Politisch wäre es sehr schwierig. Ich weiss nicht, wer den Stift halten würde', wenn es darum gehe, nationale $\mathrm{CO}_{2}$-Budgets festzulegen.

(St. Galler Tagblatt 11.11.2013) 
Das Schema (7) stellt das zugrundeliegende Argument der kognitiven Autorität dar. Eine mögliche Überführung in Practical Reasoning könnte (8) darstellen. Dies wird allerdings (zumindest teilweise) von der administrativen Autorität zurückgewiesen, wie das Zitat von Christiana Figueres zeigt. Die vierte kritische Frage (cf. Walton/Reed/Macagno 2008: 323) wird vollständig negiert, indem erklärt wird, dass sich kein Staat auf ein $\mathrm{CO}_{2}$-Reduktion einliesse. Das Expert*innenwissen gipfelt dadurch nicht in konkreten Handlungsanweisungen, sondern wird an dieser Stelle auf Grundlage der kritischen Fragen des Practical Reasoing verworfen.

Die Zusammenarbeit der beiden Autoritäten wird in Zeitungsartikeln teilweise auch als konfliktträchtig beschrieben:

Klimaforschung und Klimapolitik sind längst dabei, sich auseinanderzuleben. Die Gründe dafür sind vielfältig, aber sie lassen sich auf einen gemeinsamen Nenner bringen: Eine im umfassenden Sinne wissenschaftsbasierte Klimapolitik ist illusorisch. Klimapolitiker nehmen für ihre Pläne zwar gerne eine wissenschaftliche Legitimation in Anspruch. Im praktischen Handeln jedoch beharren sie stets auf ihrer Autonomie.

(NZZ am Sonntag 29.09.2013)

Die Diskrepanz wird verstärkt, wenn wie in (7) und ( $\left.8^{*}\right)$ die kognitive Autorität eine Handlungsempfehlung ausspricht, die von der administrativen Autorität verworfen wird. Der Anschein, dass die beiden Autoritäten gegeneinander arbeiten, wird auch an anderer Stelle anlässlich der offiziellen Versammlung mit Regierungsvertreter*innen erweckt: „Und nun gilt es in dieser Woche im Plenum, die Delegierten von 195 Regierungen Satz für Satz von den neuen Resultaten zu überzeugen“ (Der Bund 24.09.2013). Diese Beobachtung wird dadurch verstärkt, dass die Bevölkerung primär durch die administrative Autorität betroffen ist. Handelt diese kontradiktorisch zum eigentlichen Expert*innenwissen, liegt dessen Zurückweisung nahe.

Diese beiden Autoritäten und die dazugehörigen Argumentationsschemata führen nicht nur dazu, dass das Expert*innenwissen der kognitiven Autorität zurückgewiesen wird, sondern auch dazu, dass Konklusionen jeweils ,doppelt“ validiert werden: Zuerst müssen sie als Argument from Expert Opinion validiert werden, um anschliessend denselben Prozess als Practical Reasoning noch einmal zu durchlaufen.

\subsubsection{Akteur*innenbasierte Schemata}

Die Akteur*innen selbst werden nicht nur zur Legitimation im Rahmen von Autoritätsschemata verwendet, sondern ihrerseits in Argumenten diskutiert:

\section{(9) Argument from Inconsistent Commitment}

Eine Autorin des IPCC-Berichtes war auch Teil einer politischen Delegation.

(Klimawissenschaftler*innen sollen unabhängig von der Politik sein.)

Die Klimawissenschaftlerin ist abhängig von der Politik.

Die Leiterin der Gruppe für den technischen Support der Arbeitsgruppe I der Universität Bern war damals Mitglied der deutschen Delegation. Der profilierte Berner Klimaforscher Thomas Stocker - damals führender Leadautor, heute Co-Vorsitzender der Arbeitsgruppe I - war irritiert. In Paris sagt er: „Diese Vermischung von Kompetenzen ist unangebracht und nach aussen ein schlechtes Zeichen. Es könnte die Unabhängigkeit der Wissenschaft im IPCC infrage stellen. Dazu gibt es aber keinen Grund.“ 
(Tages-Anzeiger 21.09.2013)

Nicht nur Meinungen und Argumente der Akteur*innen werden diskutiert, sondern auch ihre Glaubwürdigkeit. Dies führt soweit, dass unter Umständen sogar die ursprünglichen, wissenschaftlichen Propositionen zurückgewiesen werden. Im Korpus lässt sich aber ebenso das andere Extrem finden; der IPCC wird als renommierte Institutionen etabliert und seine Schweizer Mitglieder werden dazu verwendet, die Schweiz selbst zu profilieren:

\section{(10) Argument from the Group and its Members: Variant 1}

Schweizer Expert*innen arbeiten seit Jahren intensiv an der Dokumentation mit.

(Wenn Schweizer Expert*innen einen wichtigen Beitrag in den Klimawissenschaften leisten, so leistet die Schweiz ebenfalls einen wichtigen Beitrag.)

Die Schweiz ist führend bei den Weltklimaberichten.

Schweiz führend bei Weltklimabericht; Hiesige Wissenschaftler arbeiten seit Jahren intensiv an der Dokumentation mit

(Titel eines Artikels der Basler Zeitung vom 27.09.2013)

Ebenfalls lassen sich Wendungen wie „Thomas Stocker, Professor an der Universität Bern, gehört zu den renommiertesten Klimaforschern weltweit." (Neue Zürcher Zeitung 01.11.2013) häufig antreffen. Die Universität Zürich wird 7-, die ETH Zürich 21- und die Universität Bern 32-mal in den Artikeln erwähnt. Hochschulen anderer Länder werden kaum genannt, obwohl der IPCC ein internationales Forscherkollektiv darstellt. Eine solche einseitige Berichterstattung gewichtet Schweizer Forscher*innen ungleich stärker. In einem pars-pro-toto-Schluss führt dies wie in Beispiel (10) dazu, dass die Schweiz als besonders zentrale Akteurin in den Klimawissenschaften verstanden wird. Weitere Aussagen untermauern die Bedeutung der Schweiz auch aktiv: „Der Schweizer [= Thomas Stocker] setzte sich in der direktdemokratischen Ausmarchung durch, auch weil Bundesrat Moritz Leuenberger angeboten hatte, die IPCC-Zentrale in Bern mit jährlich 1,8 Millionen Franken zu unterstützen“ (Die Weltwoche 03.10.2013).

Eigenschaften von Mitgliedern auf eine Gruppe zu übertragen, ist ein häufig anzutreffender Topos, der durch die starke Abstraktion (cf. Perelman/Olbrechts-Tyteca 1958/2004: 456) fehlschlüssig sein kann: Das Prestige einzelner Personen wie Thoma Stocker wird auf Institutionen und dann auf das ganze Land übertragen. Dass dieses Argument nur bedingt gültig ist, zeigt sich, wenn die Bevölkerung selbst thematisiert wird (erstes Zitat) oder aber sich Expert*innen zu Wort melden (zweites Zitat):

Zwar sorgt sich die Bevölkerung immer noch stark um den Klimawandel, wie Umfragen zeigen - aber der Trend zeigt klar nach unten. Vor kurzem wurde etwa bekannt, dass 39 Prozent der Deutschen die globale Erwärmung fürchten. Vor sieben Jahren waren es noch 62 Prozent. Auch in der Schweiz nimmt die Aufmerksamkeit ab.

(Die Weltwoche 03.10.2013)

Obwohl die Schweiz dank dem Projekt zwei Klimaforschungs-Institutionen hat, das Oeschger Centre for Climate Change Research an der Universität Bern und das Center for Climate Systems Modeling an der ETH Zürich, ist dieser Geldfluss jetzt versiegt. Da 
andere Länder massiv in solche Forschungsfragen investierten, sieht Stocker die internationale Stellung der Schweiz in der Klimaforschung bedroht: "Wir betrachten das mit grosser Sorge."

(Basler Zeitung 27.09.2013)

Mit dem Argument from the Group and its Members wird nicht nur die Schweiz als kognitive Autorität profiliert, sondern es werden auch andere Staaten thematisiert. Insbesondere die Frage nach der Schuld ist als Topos häufig anzutreffen:

\section{(11) Argument from the Group and its Members: Variant 2}

Staaten müssen ihre Emissionen reduzieren.

a) (Schwellenländer tragen einen Grossteil der Schuld an den momentanen $\mathrm{CO}_{2}$-Emissionen.)

b) (Aufstrebende Wirtschaftsmächte wie China und Brasilien tragen einen Grossteil der Schuld an den momentanen $\mathrm{CO}_{2}$-Emissionen.)

c) (Die USA tragen einen Grossteil der Schuld an den momentanen $\mathrm{CO}_{2}$-Emissionen.)

d) Industriestaaten tragen eine historische Schuld.

(Die Schuldigen sind dazu verpflichtet, ihren Teil dazu beizutragen, dass die Emissionen reduziert werden. Dies trifft auch auf a) bis d) zu.)

a) bis d) müssen sich dazu verpflichten, ihre Emissionen zu reduzieren.

In den zähen Verhandlungen um einen neuen UNO-Klimavertrag ist vorgesehen, dass auch Schwellenländer ihre Emissionen reduzieren müssen. In der abgelaufenen Vereinbarung, dem Kyoto-Protokoll, waren nur Industriestaaten dazu verpflichtet. Die Verteilung der Reduktionspflichten gehört zu den umstrittensten Verhandlungspunkten.

(Tages-Anzeiger 26.04.2014)

Es geht um die Tragweite der historischen Schuld der Industrieländer. Schwellenländer wie China oder Indien halten diese den Industriestaaten immer wieder vor, wenn es darum geht, global die Emissionen der Treibhausgase zu reduzieren oder finanzielle Unterstützung seitens der reichen Länder für eine nachhaltige wirtschaftliche Entwicklung einzufordern.

(Der Bund 27.09.2013)

Die kognitiven und administrativen Autoritäten des Diskurses dienen nicht nur dazu, Konklusionen zu stützen, wie in den Kapiteln 4.3.2 und 4.3.3 gezeigt wurde, sondern sie werden ihrerseits zu strittigen Konklusionen, wie die Beispiele (9) bis (11) gezeigt haben.

\section{$5 \quad$ Fazit}

Die mediale Berichterstattung über die Summaries for Policymakers beschränkt sich auf einige bestimmte Punkte der Zusammenfassungen, wobei der Fokus auf den Themen der ersten Arbeitsgruppe liegt. Dies ist unter anderem daran ersichtlich, dass ein Grossteil der Artikel im Anschluss an die Vorstellung des ersten Teilberichts erschienen. Diese Tendenz lässt sich auch an den zu analysierenden Mehrworteinheiten erkennen. Einige von ihnen betreffen lediglich die erste Arbeitsgruppe wie beispielsweise Band eins des, im ersten Teil, der Arbeitsgruppe I und auch andere lassen sich mit den Berichten der ersten Arbeitsgruppe in Verbindung bringen (z B. von 18 bis 59 Zentimetern oder in den letzten 15 Jahren). Die verhandelten Propositionen 
im Diskurs lassen sich im Wesentlichen auf die in 4.2 genannten Makrostrukturen reduzieren. Diese Reduktionen findet sowohl in klimawandelkritischen als auch in neutralen Texten statt. Zentral sind auch Abweichungen von den Modellen sowie Unterschiede zu dem Bericht der vierten Arbeitsgruppe im Jahr 2007; oft thematisiert wurden die letzten fünfzehn Jahre, deren schwacher Temperaturanstieg insbesondere in klimawandelskeptischen Texten als Argument gegen den Klimawandel aufgeführt wird. Zu diesem Problem bezieht auch Thomas Stocker im Rahmen von Interviews Stellung (z. B. Neue Luzerner Zeitung 11.01.2014).

Diese (teilweise) aus den IPCC-Berichten stammenden Propositionen werden oft im Rahmen von Argumentationsschemata (unter Umständen auch unter Berücksichtigung der kritischen Fragen) entweder als implizite oder explizite Prämissen oder als Konklusionen aufgegriffen. Aussagen der SPM bieten sich aus methodischen Gründen (s. 4.1) als strittige Konklusionen an, da sie die gleichen sprachlichen Indikatoren für die Beurteilung der wissenschaftlichen Aussagen verwenden, welche oft auch zur alltäglichen Realisierung epistemischer Modalität verwendet werden. Das ist beispielsweise daran ersichtlich, dass häufig auf kritische Fragen der einzelnen Argumentationsschemata eingegangen wird, nicht zuletzt auch im Rahmen von Expert*inneninterviews. Dieser Expert*innenbezug ist auch notwendig, da Lai*innen nur schwer in der Lage sind, Konklusionen der Spezialdiskurse beurteilen zu können.

Die Mehrworteinheiten aus Kapitel 4.2 weisen darauf hin, dass stark personen- und institutsbezogen argumentiert wird. Oft werden Aussagen über das Expert*innenwissen gesichert. „, neuste Bericht des UNO-Wissenschaftsrats macht deutlich, dass der Mensch als Hauptfaktor die Klimaerwärmung verursacht" (Neue Luzerner Zeitung 11.01.2014; Hervorhebung durch die Autorin). Diese Expert*innenstruktur sichert oft grundlegende, kausale Argumentationsstrukturen der Wissenschaft.

Problematisch an solchen Kausalschemata ist allerdings, dass ein wissenschaftlicher auf einen alltagslogischen Kausalitätsbegriff trifft. Die vorherrschende Polykausalität in den Berichten (cf. Stocker et al. 2013: 13-14) wird auf eine Monokausalität (zum Beispiel „CO-Emissionen führen zum Abschmelzen von Gletschern") und Argumentationsketten werden auf einzelne Schemata reduziert. Dadurch werden verschiedene Wechselwirkungen übergangen, was dazu führt, dass die verwendeten Kausalschemata nicht abbilden, was die Summaries for Policymakers tatsächlich beinhalten. Dadurch lassen sich die kritischen Fragen nicht mehr ausreichend beantworten. Expert*innen wirken dieser Problematik teilweise entgegen, indem sie Interviews geben oder zitiert werden. Durch die direkte Befragung der Expert*innen werden so die monokausalen Schemata entweder wieder auf polykausale Argumentationsketten erweitert oder aber deren kritische Fragen beantwortet:

Hier spielen verschiedene Faktoren zusammen, unter anderem auch die natürliche Variabilität des Klimasystems. Unsere Hauptaussagen werden dadurch aber nicht relativiert. Dass wir eine klare Erwärmung haben und diese eindeutig mit dem Einfluss des Menschen zu tun hat: Da haben wir robuste Resultate.

(Thomas Stocker in der Neue Luzerner Zeitung 11.01.2014)

Die praktische Umsetzung erfolgt dann in einem zweiten Schritt durch Staaten und Politiker*innen als administrative Autoritäten mittels Practical Reasoning. Die Konklusionen müssen die Argumentation entsprechend mehrfach durchlaufen, ehe sie in eine praktische Umsetzung auf 
der Grundlage von akzeptierten Wissen resultieren können: Zuerst werden wissenschaftliche (kausale) Argumentationen (z. B. (1) bis (5)) über eine kognitive Autorität legitimiert (z. B. (6) und (7)). Die so validierten Konklusionen werden dann mittels Practical Reasoning (z. B. (8)) von administrativen Autoritäten in eine Handlung überführt. Bei beiden Schritten scheitert die Überführung der Standpunkte teilweise an den Akteur*innen selbst (z. B. (8)) oder an institutionellen Besonderheiten (s. Kapitel 4.1), ebenfalls werden die Akteur*innen selbst zu strittigen Konklusionen (z. B. (9) bis (11)).

Aufgrund des diskursiven Aufbaus ergeben sich so zahlreiche Schwierigkeiten, die einerseits den institutionellen Besonderheiten des Diskurses und andererseits dem Verhältnis zwischen alltäglicher und wissenschaftlicher Argumentationskultur geschuldet sind. Diese Punkte führen dazu, dass die Argumentationen im printmediale Diskurs nicht selten ambivalenter und strittiger wahrgenommen werden als in den Spezialdiskursen.

Die Verwendung von Funktionswörtern als Ausgangspunkt für eine Analyse im Sinne des Diskurshistorischen Ansatzes scheint mindestens für diesen Diskurs sinnvoll zu sein. Durch den explorativen Zugang war es möglich, Argumentationsschemata unvoreingenommen zu betrachten, zentrale Schwierigkeiten in der Vermittlung zu erkennen und auch im institutionellen Rahmen zu verorten. Durch die gezielte Auswahl von bestimmten Funktionswörtern (insbesondere in Hinblick auf potentielle Argumentationsindikatoren) wäre es unter Umständen möglich, gezielter spezifische Schemata im Diskurs zu elaborieren: Dies müsste aber in einem nächsten Schritt evaluiert werden.

\section{Literaturverzeichnis}

Besio, Cristina/Pronzini, Andrea (2010): „Unruhe und Stabilität als Form der massenmedialen Kommunikation über Klimawandel“. In: Voss, Martin (ed.): Der Klimawandel. Sozialwissenschaftliche Perspektive. Wiesbaden, VS Verlag für Sozialwissenschaften: 283-299.

Boykoff, Maxwell T. (2011): Who Speaks for the Climate? Making Sense of Media Reporting on Climate Change. Cambridge: Cambridge University Press.

Bubenhofer, Noah (2008): ,,Es liegt in der Natur der Sache... '. Korpuslinguistische Untersuchungen zu Kollokationen in Argumentationsfiguren“. In: Mellado Blanco, Carmen (ed.): Beiträge zur Phraseologie aus textueller Sicht. Hamburg, Kovač: 53-72. (= PHILOLOGIA - Sprachwissenschaftliche Forschungsergebnisse 112).

Bußmann, Hadumod (2002): Lexikon der Sprachwissenschaft. 4., aktualisierte und erweiterte Auflage. Stuttgart: Kröner.

Edenhofer, Ottmar et al. (eds.) (2014): "IPCC, 2014. Summary for Policymakers". In: idd.: Climate Change 2014, Mitigation of Climate Change. Contribution of Working Group III to the Fifth Assessment Report of the Intergovernmental Panel on Climate Change. Cambridge/New York, Cambridge University Press: 1-31. http://www.ipcc.ch/pdf/assessmentreport/ar5/wg3/ipcc_wg3_ar5_summary-for-policymakers.pdf [31.10.2017].

Ehrensperger, Anna (2009): Klimawandel medial. Die Klimawandelberichterstattung in der Deutschschweizer Tagespresse vor dem Hintergrund des medialen Informationsbias. Hamburg: Academic Transfer. 
Eemeren, Frans H. van/Grootendorst, Rob (2004): A Systematic Theory of Argumentation. The Pragma-dialectical Approach. New York: Cambridge University Press.

Eemeren, Frans H. van /Houtlosser, Peter/Snoeck Henkemans, A. Francisca (2007): Argumentative Indicators in Discourse. A Pragma-dialectical Study. Dordrecht: Springer. (= Argumentation Library 12).

Field, Christopher B. et al. (eds.) (2014): "IPCC, 2014. Summary for Policymakers". In: idd.: Climate Change 2014. Impacts, Adaptation and Vulnerability. Part A: Global and Sectoral Aspects. Contribution of Working Group II to the Fifth Assessment Report of the Intergovernmental Panel on Climate Change. Cambridge, Cambridge University Press: 1-32. http://www.ipcc.ch/pdf/assessment-report/ar5/wg2/ar5_wgII_spm_en.pdf [31.10.2017].

Groom, Nicholas (2010): "Closed-Class Keywords and Corpus-Driven Discourse Analysis". In: Bondi, Marina/Scott, Mike (eds.): Keyness in Texts. Amsterdam/Philadelphia, John Benjamins Publishing: 59-78. (= Studies in Corpus Linguistics 41).

Grundmann, Reiner (2006): “Ozone and Climate. Scientific Consensus and Leadership". In: Science, Technology, \& Human Values 31/1: 73-101.

IPCC - Intergovernmental Panel on Climate Change (ed.) (1999-2008): “Appendix A to the Principles Governing IPCC Work. Procedures for the Preparation, Review, Acceptance, Adoption, Approval and Publication of IPCC Reports". http://www.ipcc.ch/pdf/ipcc-principles/ipcc-principles-appendix-a.pdf [31.10.2017].

Kammermann, Nadine (2014): Funktionswörter als intratextueller Zugang zu transtextuellen Argumentationsstrukturen. Der Zusammenhang zwischen Funktionswörtern auf der intratextuellen und Argumentationsstrukturen auf der transtextuellen Ebene des Diskurses am Beispiel der Berichterstattung über die Summaries for Policymakers des fünften IPCC Assessment Report. Bern. Unveröffentlichte Masterarbeit.

Kienpointner, Manfred (1992): Alltagslogik. Struktur und Funktion von Argumentationsmustern. Stuttgart: Friedrich Frommann/Günther Holzboog. (= problemata 126).

Kindt, Walther (2008): „Die Rolle sprachlicher Indikatoren für Argumentationsanalysen. Ein Ergebnisbericht aus der Linguistischen Rhetorik“. In: Kreuzbauer, Günther/Gratzl, Norbert/Hiebl, Ewald (eds.): Rhetorische Wissenschaft. Rede und Argumentation in Theorie und Praxis. Wien, LIT-Verlag: 147-162.

Kopperschmidt, Josef (1989): Methodik der Argumentationsanalyse. Stuttgart: Friedrich Frommann/Günther Holzboog. (= problemata 119$)$.

Mastrandrea, Michael D. et al. (2010): "Guidance Note for Lead Authors of the IPCC Fifth Assessment Report on Consistent Treatment of Uncertainties". http://www.ipcc.ch/pdf/supporting-material/uncertainty-guidance-note.pdf [31.10.2017].

Neverla, Irene/Schäfer, Mike S. (2012) (eds.): Das Medien-Klima. Fragen und Befunde der kommunikationswissenschaftlichen Klimaforschung. Wiesbaden: VS Verlag für Sozialwissenschaften.

Oakes, Michael P. (1998): Statistics for Corpus Linguistics. Edinburgh: Edinburgh University Press. (= Edinburgh Textbooks in Empirical Linguistics 3).

Ottmers, Clemens (2007): Rhetorik. Überarbeitet von Fabian Klotz. 2., aktualisierte und erweiterte Auflage. Stuttgart/Weimar: J. B. Metzler. (= Sammlung Metzler 283).

Pearce, Michael (2014): "Key Function Words in a Corpus of UK Election Manifestos". Linguistik online 65/3: 23-44. 
Perelman, Chaim/Olbrechts-Tyteca, Lucie (1958/2004): Die neue Rhetorik. Eine Abhandlung über das Argumentieren. 2 Bände. Stuttgart: Friedrich Frommann/Günther Holzboog. (= problemata 149).

Reisigl, Martin (2011): «Grundzüge der Wiener Kritischen Diskursanalyse». In: Keller, Reiner et al. (eds.): Handbuch Sozialwissenschaftliche Diskursanalyse. Teil 1: Theorien und Methoden. 3. Auflage. Wiesbaden, VS Verlag für Sozialwissenschaften: 459-497.

Reisigl, Martin (2014): “Argumentation Analysis and the Discourse-Historical Approach. A Methodological Framework". In: Hart, Christopher/Cap, Piotr (eds.): Contemporary Critical Discourse Studies. London, Bloomsbury Academic: 67-96.

Reisigl, Martin/Wodak, Ruth (2016): "The Discourse-Historical Approach (DHA)". In: Wodak, Ruth/Meyer, Michael (eds.): Methods of Critical Discourse Analysis. 3. Auflage. Los Angeles, Sage: 23-61.

"Review of IPCC Processes and Procedures". http://ipcc.ch/organization/organization_review.shtml [08.07.2016].

Schmidt, Andreas/Ivanova, Ana/Schäfer, Mike S. (2013): "Media Attention for Climate Change around the World. A Comparative Analysis of Newspaper Coverage in 27 Countries". Global Environmental Change 23/5: 1233-1248.

Steinseifer, Martin (2005): „Die Sloterdijk-Debatte. Linguistische Diskursanalyse eines (Print-)Medienereignisses und seiner argumentativen Ordnung“. In: Wengeler, Martin (ed.): Sprachgeschichte als Zeitgeschichte. Konzepte, Methoden und Forschungsergebnisse der Düsseldorfer Sprachgeschichtsschreibung für die Zeit nach 1945. Hildesheim/New York, Olms: 498-526. (= Germanistische Linguistik 180-181).

Stocker, Thomas F. et al. (eds.) (2013): „IPCC, 2013. Summary for Policymakers“. In: idd.: Climate Change 2013. The Physical Science Basis. Contribution of Working Group I to the Fifth Assessment Report of the Intergovernmental Panel on Climate Change. Cambridge/New York, Cambridge University Press: 3-29. http://www.ipcc.ch/pdf/assessmentreport/ar5/wg1/WG1AR5_SPM_FINAL.pdf [31.10.2017].

Walton, Douglas N. (1997): Appeal to Expert Opinion. Arguments from Authority. Pennsylvania: Pennsylvania State University Press.

Walton, Douglas N./Reed, Chris/Macagno, Fabrizio (2008): Argumentation Schemes. Cambridge: Cambridge University Press.

Wengeler, Martin (2003): Topos und Diskurs. Begründung einer argumentationsanalytischen Methode und ihre Anwendung auf den Migrationsdiskurs (1960-1985). Tübingen: Niemeyer. (= Reihe Germanistische Linguistik 244).

Wodak, Ruth et al. (2009): The Discoursive Construction of National Identity. 2. Auflage. Edinburgh: Edinburgh University Press.

"Working Group I: The Physical Science Basis". https://wg1.ipcc.ch [31.10.2017].

"Working Group II: Impacts, Adaptation, and Vulnerability". http://www.ipcc-wg2.awi.de, [31.10.2017].

"Working Group III: Mitigation of Climate Change, about us". https://www.ipccwg3.ac.uk/aboutus.html [31.10.2017]. 


\section{Verwendete Zeitungsartikel ${ }^{10}$}

„Arbeit an neuem Klimavertrag“. St. Galler Tagblatt 11.11.2013.

„Der Klimaforscher mit der dicken Haut; Der Umweltphysiker Thomas Stocker sieht sich nicht als Aktivist - schliesst aber nicht aus, einmal einer zu werden“. Neue Zürcher Zeitung 01.11.2013.

„Der Weltklimarat behält den Überblick“. Tages-Anzeiger 21 .09.2013.

„Die Schweiz ist führend beim Weltklimabericht“. St. Galler Tagblatt 27.09.2013.

„Ein Weckruf für die Politik; Festgefahrene Klimaverhandlungen - Ziel eines bindenden Abkommens noch immer in weiter Ferne“. Neue Zürcher Zeitung 28.09.2013.

„Grösste Herausforderung unserer Zeit“. Neue Luzerner Zeitung 11.01.2014.

„Handeln muss die Politik; Die wissenschaftsbasierte Klimapolitik ist gescheitert. In Zukunft wird sich ein pragmatischerer Stil durchsetzen. Für die Qualität der Wissenschaft kann das nur positiv sein“. NZZ am Sonntag 29.09.2013.

„Klimaforscher kritisieren politischen Druck der Regierungen». Tages-Anzeiger 26.04.2014.

„Kohle statt Wende“. St. Galler Tagblatt 14.04.2014

„Politische Wissenschaft“. Die Weltwoche 03.10.2013.

„Regierungen bremsen Klimaberatung“. Der Bund 27.09.2013.

„Schweiz führend bei Weltklimabericht; Hiesige Wissenschaftler arbeiten seit Jahren intensiv an der Dokumentation mit“. Basler Zeitung 27.09.2013.

„Stagnierungsphasen wird es auch in Zukunft immer wieder geben». Der Bund 24.09.2013.

„Uno-Gremium fordert mehr Energieeffizienz und neue Techniken zur Minderung des Treibhauseffekts“. Neue Zürcher Zeitung 15.04.2014.

\footnotetext{
${ }^{10}$ Das Quellenverzeichnis enthält nur diejenigen der 125 Zeitungsartikel, die im vorliegenden Artikel zitiert wurden.
} 